\title{
Response to Periodic Disturbances in the Glass Redraw Process
}

\author{
S. Marquis ${ }^{1}$, D. O'Kiely ${ }^{1}$, P. D. Howell ${ }^{1}$, \\ U. Lange ${ }^{2} \&$ I. M. Griffiths ${ }^{1 *}$
}

Received: date / Accepted: date

\begin{abstract}
The redraw process is a method employed for the manufacture of glass sheets required for e.g. special optical filters, bendable displays, or wearable devices. During this process, a glass block is fed into a heater zone and drawn off to reduce its thickness. Fluctuations in the feed speed, the draw speed or the ambient temperature can all lead to irregularities in the final thickness profile. We present a linearized theory that allows the response to any given fluctuation to be computed, and obtain a simplified model in the high-frequency limit. The resulting framework allows for fast and efficient parameter sweeps that determine the most dangerous frequencies to be avoided and provide an important complementary tool for experimentalists working in the redraw process.
\end{abstract}

Keywords Glass manufacture - Redraw process · Asymptotic analysis · Linearized theory

\section{Introduction}

Specialty glasses, such as glasses with a very high refractive index, are usually prone to devitrification. Hence, common sheet glass forming processes such as float, or overflow fusion, are not suited for these glasses. The glass redraw process is an attractive option to manufacture thin sheets of specialty glasses, which are becoming more and more attractive for special optical filters, bendable displays or wearable devices $[3,9]$. In the redraw process, a prefabricated slab of glass is fed into a heating zone, where it melts. A thin glass sheet is then drawn out of the end of the heating zone at a higher speed, as illustrated in Figure 1. The larger the draw ratio between the draw and feed speeds, the

\footnotetext{
${ }^{1}$ Mathematical Institute, Radcliffe Observatory Quarter, Woodstock Road, Oxford, OX2 $6 \mathrm{GG}$

${ }^{2}$ Schott AG, Hattenbergstrasse 10, 55122 Mainz, Germany

E-mail: *ian.griffiths@maths.ox.ac.uk
} 


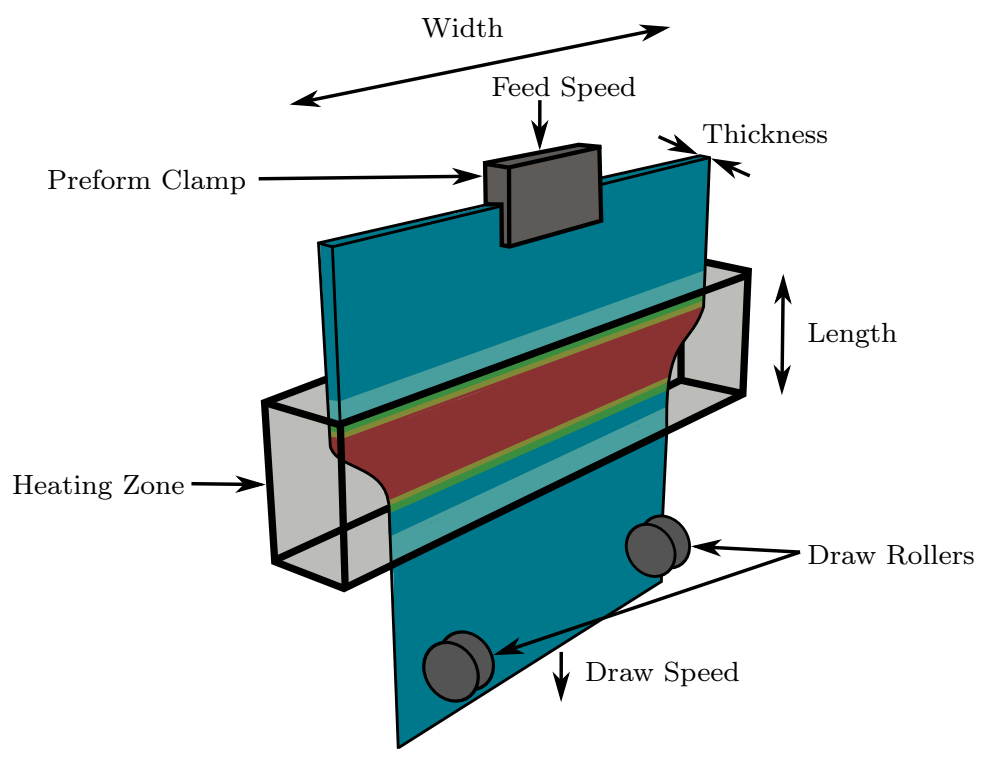

Fig. 1: Schematic of the glass redraw process.

thinner the final product. The importance of the redraw process and drawing processes in general has motivated research on a variety of sub-problems such as the role of heat transfer in the drawing process $6,13,12,23$, thickness variation in drawn/redrawn sheets $[5,4,22,7,15$, and stability of the drawn process.

Redraw is a batch process, but each batch runs for a long time so that it effectively operates in steady state, as is essential for the manufacture of glass sheets with uniform thickness. Above a certain draw ratio, a stretched viscous sheet or fibre becomes unstable to infinitesimal perturbations, a phenomenon known as draw resonance [17, 8, 11, 18, 19. Studies on draw resonance have included investigating temperature effects [13, 20, 21, investigating viscoelastic effects [22, and attempts to explain the physical cause of the phenomenon through kinematic wave theory 11. Even below the critical draw ratio, small fluctuations in the feed speed, draw speed, and air temperature within the furnace can lead to thickness variations in the final product, whose amplitude

depends on the frequency of the disturbance. If the most problematic disturbance modes are identified then they can be avoided by employing control systems, so that non-uniformities in the final product are reduced to an acceptable level.

Full computational fluid dynamics (CFD) simulations may be conducted to identify the response to different disturbances in the redraw process. However, extremely fine resolution is required to obtain reliable results, and it is time consuming to compute solutions across a wide range of parameter values and 


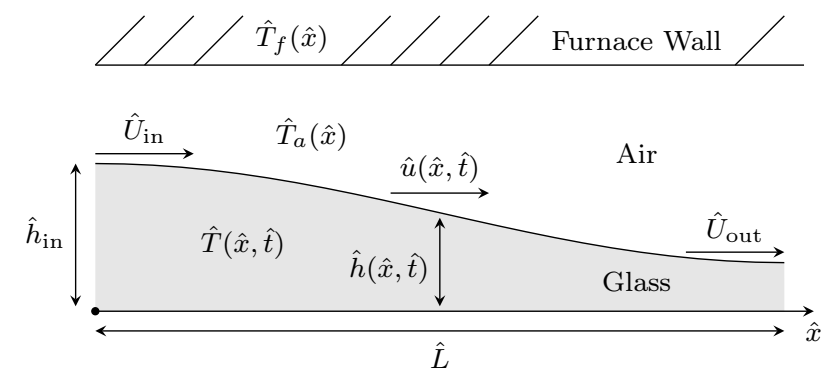

Fig. 2: Schematic of the redraw process. The $\hat{x}$-axis is a line of symmetry.

frequencies. Instead, we exploit the fact that in redraw the sheet thickness is small in comparison to its length, and consequently the glass undergoes extensional flow, with its lengthwise velocity approximately uniform across its thickness. This allows us to describe the system using a reduced quasi-onedimensional model $10,12,24$. We thus propose and implement asymptotically reduced mathematical models and fast numerical schemes to predict the response to different disturbance modes over the entire spectrum of frequency perturbations.

In $\S 2$, we outline our mathematical model, and we determine the steadystate solution in $\$ 3$. We perform a linear-stability analysis in 84 , and determine how the response to different disturbances depends on process parameters in $\$ 5$ In 86 we focus on high-frequency perturbations. Finally, in $\$ 7$ we draw conclusions from the results that we have presented and the strategy for implementation in the glass industry.

\section{Mathematical model}

We consider a glass preform of thickness $2 \hat{h}_{\text {in }}$ that enters a heating zone of length $\hat{L}$ at speed $\hat{U}_{\text {in }}$, and is drawn from the end of the heating zone at speed $\hat{U}_{\text {out }}$, as illustrated in Figure 2. Throughout this paper, dimensional quantities will be indicated by the presence of a 'hat'. We denote the distance along the heating zone by $\hat{x} \in[0, \hat{L}]$, the temperature of the furnace wall by $\hat{T}_{f}(\hat{x})$, and the air temperature in the heating zone by $\hat{T}_{a}(\hat{x})$. When the rate of heat transfer between the atmosphere and the glass (as measured by the Biot number) is sufficiently large, significant cross-sectional variations in temperature may occur [20]. However, as mentioned in the Introduction, when the sheet is sufficiently thin, the velocity $\hat{u}$ in the $\hat{x}$-direction is approximately uniform across the thickness of the sheet, and similarly the temperature $\hat{T}$ is approximately uniform across the thickness. Therefore the half-thickness of the glass, $\hat{h}(\hat{x}, \hat{t})$, the speed of the glass $\hat{u}(\hat{x}, \hat{t})$, and the temperature of the glass $\hat{T}(\hat{x}, \hat{t})$ are to be determined in the heating zone. 
We express our system in dimensionless form by employing the scalings

$$
\begin{aligned}
& \hat{x}=\hat{L} x, \quad \hat{t}=\frac{\hat{L}}{\hat{U}_{\text {in }}} t, \quad \hat{T}_{f}(\hat{x})=\hat{T}_{s} T_{f}(x), \quad \hat{T}_{a}(\hat{x})=\hat{T}_{s} T_{\mathrm{a}}(x), \\
& \hat{h}(\hat{x}, \hat{t})=\hat{h}_{\text {in }} h(x, t), \quad \hat{u}(\hat{x}, \hat{t})=\hat{U}_{\text {in }} u(x, t), \quad \hat{T}(\hat{x}, \hat{t})=\hat{T}_{s} T(x, t),
\end{aligned}
$$

where the lack of a 'hat' indicates a dimensionless quantity. Here, $\hat{T}_{s}$ is a characteristic temperature of the glass.

We identify a small aspect ratio $\delta=\hat{h}_{\text {in }} / \hat{L}$ and exploit the asymptotic limit $\delta \rightarrow 0$ to obtain a reduced description of the problem: the Trouton model. A full derivation may be found in 10,14 ; here we just present the resulting leading-order equations, valid to order $\delta^{2}$. We note in passing that variations in the velocity field could be considered by extending to higher-order terms in a similar manner as considered by 16 . Conservation of mass, momentum and heat energy in the glass leads respectively to the equations

$$
\begin{aligned}
& h_{t}+(u h)_{x}=0, \\
& 4 \mu h u_{x}=F(t), \\
& h\left(T_{t}+u T_{x}\right)=-a\left[\left(T^{4}-T_{f}^{4}\right)+b\left(T-T_{a}\right)\right],
\end{aligned}
$$

where, $\mu=\mu(T)$ is the dimensionless viscosity of glass and $F$ is the dimensionless tension in the sheet (to be determined as part of the solution). The dimensionless parameters

$$
a=\frac{\hat{L} \hat{\sigma} \hat{\epsilon}_{r} \hat{T}_{s}^{3}}{\hat{h}_{\mathrm{in}} \hat{\rho} \hat{c}_{p} \hat{U}_{\mathrm{in}}}, \quad b=\frac{\hat{m}_{h}}{\hat{\sigma} \hat{\epsilon}_{r} \hat{T}_{s}^{3}},
$$

are respectively the radiative Stanton number and ratio of the Stanton number to the radiative Stanton number; $\hat{\rho}, \hat{c}_{p}$ and $\hat{\epsilon}_{r}$ are the density, specific heat capacity and specific emissivity of the glass, $\hat{\sigma}$ is the Stefan-Boltzmann constant, and $\hat{m}_{h}$ is the heat-transfer coefficient. The Stanton number and the radiative Stanton number measure the rates of heat transfer to the glass via the air and via radiation from the furnace walls, respectively, relative to the thermal capacity of the glass.

At the top of the heater zone, the glass is fed in with unit dimensionless speed and half-thickness, and we assume the temperature is equal to that of the furnace wall at the inlet, so

$$
u(0, t)=1, \quad h(0, t)=1, \quad T(0, t)=T_{f}(0) .
$$

At the bottom of the heater zone, the glass is drawn at a fixed speed

$$
u(1, t)=D
$$

where $D=\hat{U}_{\text {out }} / \hat{U}_{\text {in }}$ is the draw ratio. 


\begin{tabular}{cc}
\hline Symbol & Typical Value \\
\hline$D$ & 25 \\
$a$ & 3.7 \\
$b$ & 0.022 \\
$\nu$ & 3.9 \\
$T_{\mathrm{c}}$ & 0.66 \\
\hline
\end{tabular}

\begin{tabular}{cc}
\hline Symbol & Typical Value \\
\hline$\theta_{0}$ & 0.70 \\
$\theta_{1}$ & 0.30 \\
$x_{0}$ & 0.25 \\
$k$ & 0.063 \\
$\bar{T}_{a}$ & 0.75 \\
\hline
\end{tabular}

Table 1: Typical industrially relevant dimensionless parameter values [1].

To close the model, we must impose a constitutive relation for the glass viscosity and also specify the furnace wall and air temperatures. In this paper we take the following representative functional forms:

$$
\begin{aligned}
\mu(T) & =\exp \left(\nu\left(\frac{1}{T-T_{c}}-\frac{1}{1-T_{c}}\right)\right), \\
T_{f}(x) & =\theta_{0}+\theta_{1} \exp \left(-\frac{\left(x-x_{0}\right)^{2}}{k^{2}}\right), \\
T_{a}(x) & =\bar{T}_{a} T_{f}(x)
\end{aligned}
$$

where $\nu, T_{c}, \theta_{0}, \theta_{1}, x_{0}, k$, and $\bar{T}_{a}$ are empirically determined constants. A set of industrially relevant dimensionless parameter values is displayed in Table 1 For this parameter set, the air temperature in the furnace is below that of the furnace wall. Therefore, since the input glass temperature (2e) is taken to be equal to the temperature of the furnace wall, the glass will initially cool upon entrance into the furnace. Additionally, the furnace air temperature is below $T_{c}$, so if the heat exchange with the air relative to the heat exchange with the furnace wall (controlled by the parameter $b$ ) is too large the glass will reach the temperature $T_{c}$ and our viscosity model (3a) breaks down. We choose to limit our study to values of $b$ where this does not occur to demonstrate our results but note that $(3 \mathrm{a})$ can be modified if required to describe the viscosity behaviour at temperatures below $T_{c}$.

\section{Steady-state solution}

We first seek a steady-state solution to (2), of the form

$$
u(x, t)=u_{\mathrm{eq}}(x), \quad h(x, t)=h_{\mathrm{eq}}(x), \quad T(x, t)=T_{\mathrm{eq}}(x), \quad F(t)=F_{\mathrm{eq}} .
$$

From $2 \mathrm{a}$ and $(2 \mathrm{e})$, we obtain

$$
u_{\mathrm{eq}} h_{\mathrm{eq}}=1 .
$$

Combining (2c) and (2e) gives a decoupled problem for the temperature of the sheet, namely

$$
T_{\mathrm{eq}}^{\prime}=-a\left[\left(T_{\mathrm{eq}}^{4}-T_{f}^{4}\right)+b\left(T_{\mathrm{eq}}-T_{a}\right)\right], \quad T_{\mathrm{eq}}(0)=T_{f}(0),
$$


which we solve numerically. Given $T_{\text {eq }}$ from $5 \mathrm{a}$, we use $2 \mathrm{~b}, 2 \mathrm{2e}$, and $2 \mathrm{ff}$ to find

$$
\begin{aligned}
F_{\mathrm{eq}} & =\frac{4 \log (D)}{I(1)}, \\
u_{\mathrm{eq}}(x) & =\exp \left(\log (D) \frac{I(x)}{I(1)}\right), \\
h_{\mathrm{eq}}(x) & =\exp \left(-\log (D) \frac{I(x)}{I(1)}\right),
\end{aligned}
$$

where

$$
I(x)=\int_{0}^{x} \mu\left(T_{\mathrm{eq}}(\bar{x})\right)^{-1} \mathrm{~d} x
$$

The steady solution given by (5) is plotted in Figure 3 for the parameters listed in Table 1. We observe in Figure 3(a) that the glass is first heated and and then cooled by heat transfer with the furnace, with the maximum glass temperature occurring near $x \approx 0.3$. Given the smallness of $b$, heat transfer is dominated by radiation. With $a=3.7$, the glass temperature somewhat lags behind the furnace temperature, and we would expect these to become closer if the value of $a$ were increased. The air temperature $T_{a}$ is lower than the temperature of the glass when it enters the heating zone. Therefore, the glass temperature initially slightly decreases due to cooling by the air. This small effect is undetectable in Figure 3(a), but gives rise to the initial increase in the viscosity in Figure 3 (b). However, the viscosity soon starts to decrease rapidly, and the minimum viscosity occurs at the location of the maximum glass temperature. As a result, the stretching and associated thickness variations of the glass are also localized to a neighbourhood of the maximum temperature, as shown in Figure 3(c) and (d).

\section{Small-amplitude perturbations}

We now turn our attention to the effect of perturbations to the feed speed, draw speed and air temperature on the steady-state solution (5) by setting

$$
\begin{aligned}
u(0, t) & =1+\epsilon f_{\text {in }} \exp (i \omega t), \\
u(1, t) & =D\left(1+\epsilon f_{\text {out }} \exp (i \omega t)\right), \\
T_{a}(x, t) & =\bar{T}_{a}\left(T_{f}(x)+\epsilon f_{T}(x) \exp (i \omega t)\right),
\end{aligned}
$$

where $\epsilon \ll 1$ and $\omega \geq 0$ is the frequency of the perturbation. Here, we consider a representative Gaussian temperature perturbation of the form

$$
f_{T}(x)=A \exp \left(-\frac{\left(x-x_{\mathrm{loc}}\right)^{2}}{2 \sigma^{2}}\right),
$$



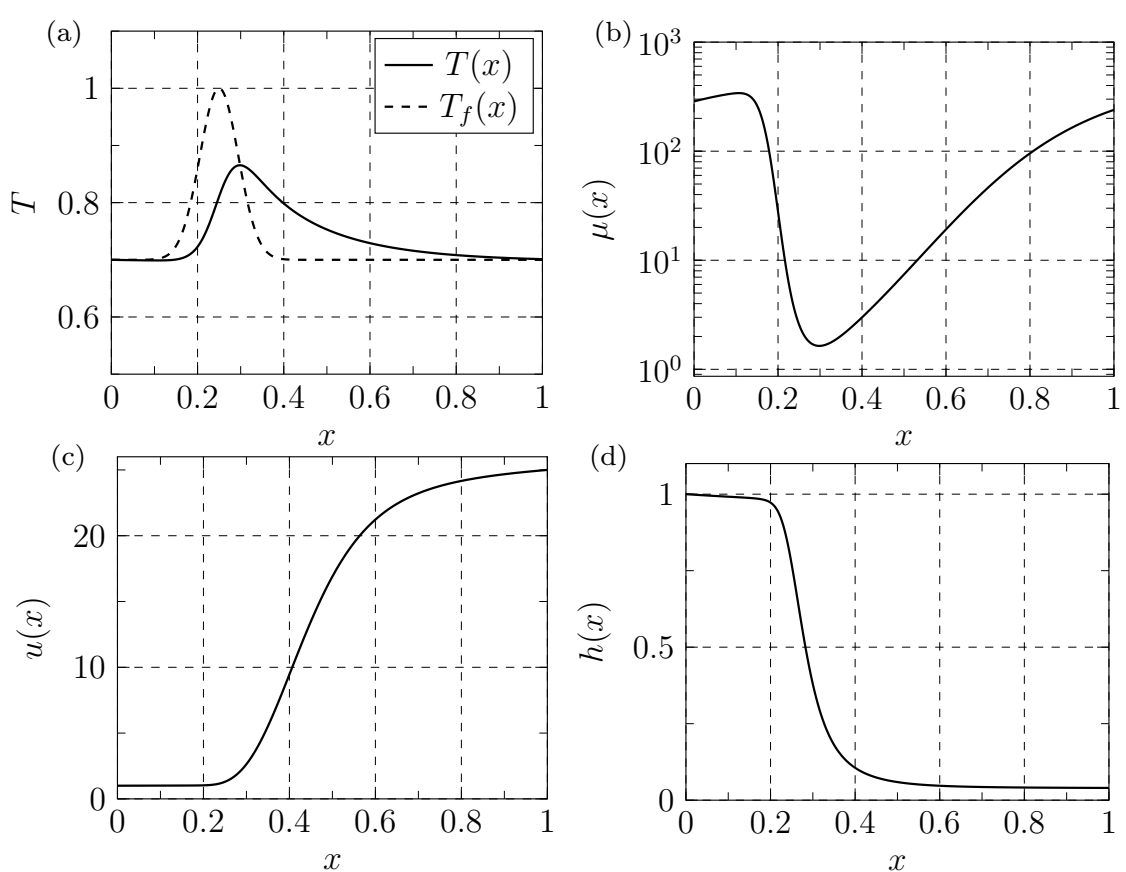

Fig. 3: Steady-state solution to 2 for the parameters in Table 1

where $x_{\text {loc }}$ is the location of the maximum perturbation to the air temperature, while $A$ and $\sigma$ control the amplitude and extent of the perturbation.

Based on our assumption that the perturbations are small, we seek a solution of the form

$$
\begin{aligned}
u(x, t) & =u_{\mathrm{eq}}(x)(1+\epsilon \tilde{u}(x) \exp (i \omega t)), \\
h(x, t) & =h_{\mathrm{eq}}(x)(1+\epsilon \tilde{h}(x) \exp (i \omega t)), \\
T(x, t) & =T_{\mathrm{eq}}(x)(1+\epsilon \tilde{t}(x) \exp (i \omega t)), \\
F(t) & =F_{\mathrm{eq}}(1+\epsilon \tilde{F} \exp (i \omega t)),
\end{aligned}
$$

for some $\tilde{u}, \tilde{h}, \tilde{t}$, and $\tilde{F}$ to be determined. We insert this ansatz into 2 and linearize with respect to $\epsilon$ to obtain

$$
\begin{aligned}
& \tilde{h}^{\prime}+\tilde{u}^{\prime}+i \omega h_{\mathrm{eq}} \tilde{h}=0, \\
& \tilde{u}^{\prime}+h_{\mathrm{eq}} u_{\mathrm{eq}}^{\prime}\left(\tilde{u}+\tilde{h}-\frac{\nu T_{\mathrm{eq}}}{\left(T_{\mathrm{eq}}-T_{\mathrm{c}}\right)^{2}} \tilde{t}-\tilde{F}\right)=0, \\
& T_{\mathrm{eq}} \tilde{t}^{\prime}+T_{\mathrm{eq}}^{\prime} \tilde{t}+T_{\mathrm{eq}}^{\prime}(\tilde{h}+\tilde{u})+a\left[4 T_{\mathrm{eq}}^{4}+b T_{\mathrm{eq}}\right] \tilde{t}+i \omega h_{\mathrm{eq}} T_{\mathrm{eq}} \tilde{t}-a b \bar{T}_{a} f_{T}=0,
\end{aligned}
$$

with boundary conditions:

$$
\tilde{u}(0)=f_{\text {in }}, \quad \tilde{h}(0)=0, \quad \tilde{t}(0)=0, \quad \tilde{u}(1)=f_{\text {out }} .
$$


In the absence of any imposed perturbations, $f_{\text {in }}=f_{\text {out }}=f_{T}=0$, and the resulting eigenvalue problem (9) admits non-trivial solutions for $\{\tilde{h}, \tilde{u}, \tilde{t}\}$ only if the (complex) frequency $\omega$ takes one of a discrete set of values. The instability known as draw resonance occurs through a Hopf bifurcation as $\operatorname{Im}[\omega]$ passes through zero (see, e.g., [20]). Here instead we impose an oscillatory disturbance either through the inhomogeneous boundary conditions $9 \mathrm{~d}$ or through the temperature perturbation $f_{T}(x)$, as defined in (7), and assess the size of the resulting oscillation in thickness. We quantify the response of the system to the imposed perturbation by defining a relative response amplitude

$$
\mathcal{R}:=\frac{\mid \text { relative variation in final thickness } \mid}{\mid \text { relative change in } \phi \mid},
$$

where $\phi$ represents one of $u(0, t), u(1, t)$ or $\int_{0}^{1} T_{a}(x, t) \mathrm{d} x$. By linearity, we can consider each perturbation in isolation and, without loss of generality, set

$$
f_{\text {in }}, f_{\text {out }}, \int_{0}^{1} f_{T}(x) \mathrm{d} x \in\{0,1\}
$$

so that

$$
\mathcal{R}=|\tilde{h}(1)|
$$

We discretize (9) using second-order backwards differences, using the steadystate solutions (5), to obtain a linear system of equations which we solve for a given frequency $\omega$ and perturbation $f_{\text {in }}, f_{\text {out }}$ or $f_{T}$.

\section{Results}

We now present numerical solutions to the perturbation equations $(9)$ and quantify the effect on the final product using the relative response $\mathcal{R}$ defined in (11). We begin by studying the isothermal case (\$5.1) and the specific industrially relevant case where the parameters are taken to be those in Table 1 (\$5.2). We then perform more in-depth parameter sweeps to characterize the response $\mathcal{R}$ for feed speed $(\$ 5.4)$, draw speed $(\$ 5.5)$ and temperature disturbances $(5.6$.

\subsection{Isothermal case}

The linear stability of the isothermal problem is analysed in 17 . It is found that the response amplitude $\mathcal{R}$ is the same for either feed or draw speed variations, and in either case is given by

$$
\mathcal{R}=\left|\frac{\operatorname{Ei}\left(\frac{i \omega}{\log D}\right)-\operatorname{Ei}\left(\frac{i \omega}{D \log D}\right)}{\exp \left(\frac{i \omega}{D}\right)-\exp \left(\frac{i \omega}{D \log D}\right)+\left(1-\frac{i \omega}{D \log D}\right)\left[\operatorname{Ei}\left(\frac{i \omega}{D}\right)-\operatorname{Ei}\left(\frac{i \omega}{D \log D}\right)\right]}\right|
$$


(a)

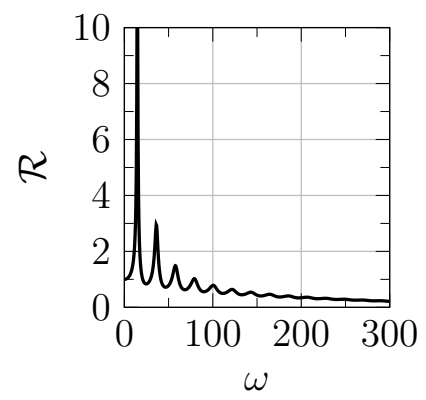

(b)

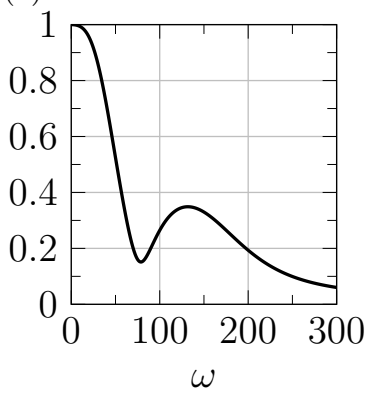

(c)

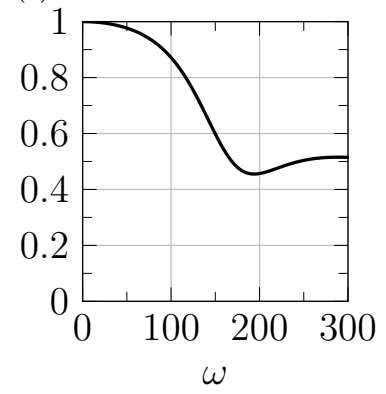

Fig. 4: Amplitude of thickness response $\mathcal{R}$, as predicted by the linearized model (9) with the parameter values listed in Table 1. (a) feed- or draw-speed disturbance in an isothermal sheet $(a=0)$; (b) feed-speed disturbance for redraw through a typical furnace; (c) draw-speed disturbance for redraw through a typical furnace.

where Ei denotes the exponential integral function. The denominator of the expression $(12)$ is zero at the critical values $(\omega, D)=(14.011,20.218)$ corresponding to the onset of draw resonance.

Figure 4(a) shows how the response amplitude given by 12 varies with fluctuation frequency $\omega$ with fixed draw ratio $D=25$. Since we are above the critical draw ratio, the steady state is linearly unstable. The system exhibits resonance, with a greatly amplified thickness variation close to the critical frequency $\omega \approx 14.011$ associated with draw resonance. There is a sequence of subsequent peaks corresponding to damped resonant modes close to the other zeros $\omega$ of the denominator of $[12$ with $\operatorname{Im}[\omega]>0$, but the response ultimately decays as the perturbation frequency tends to infinity.

\subsection{Industrially relevant regime}

We observe dramatically different behaviour for the temperature-dependent system. Here the responses to feed- and draw-speed perturbations are different, and are plotted in figures Figure 4(b) and (c), respectively. The response $\mathcal{R}$ is significantly smaller than in the isothermal case, with the largest response as $\omega \rightarrow 0$ and a second, local, maximum at $\omega \approx 130$ for a disturbance to the feed speed, and $\omega \approx 288$ for a disturbance to the draw speed. Beyond this second peak, $\mathcal{R}$ appears to decrease with increasing $\omega$, and we terminate our search at $\omega=500$.

In Figure 5, we study the response due to disturbances in the surrounding air temperature. We perform two investigations: (a) varying the frequency, while fixing the disturbance location $x_{\mathrm{loc}}=0.45$, and (b) varying the location of the disturbance, while fixing the frequency $\omega=200$. In all cases we set the spread of the temperature perturbation to $\sigma^{2}=0.001$. We observe that 


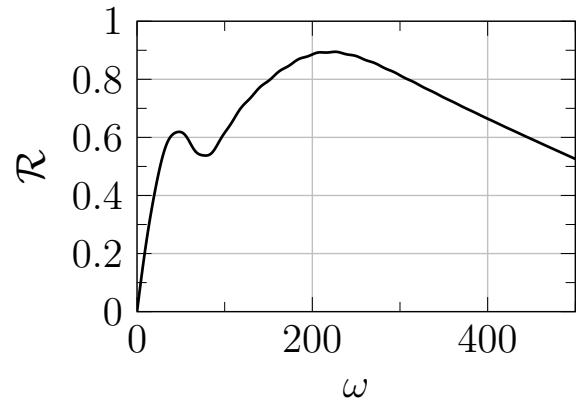

(a) $x_{\text {loc }}=0.45$

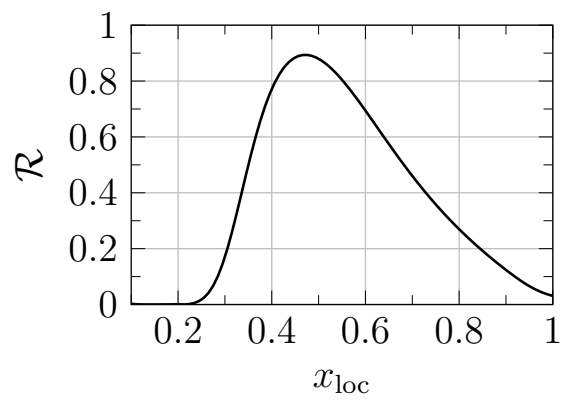

(b) $\omega=200$

Fig. 5: Amplitude of thickness response $\mathcal{R}$, as predicted by the linearized model (9) for the temperature-dependent system with $f_{\text {in }}=f_{\text {out }}=0$ and $f_{T}(x)$ in the form of 77 with $\sigma^{2}=0.001$. All other parameters are as listed in Table 1 . (a) Response dependence on frequency; (b) response dependence on location.

the peak response occurs at a frequency of $\omega \approx 210$, with a large band of surrounding frequencies that excite a similar magnitude response. Therefore, it is likely that frequencies between $150 \lesssim \omega \lesssim 300$ would need to be controlled to keep the thickness fluctuations below an acceptable level. At very low frequencies, the system can adjust to a quasi-steady state where the glass simplify deforms at a different part of the heating zone, whose location varies parametrically with time. At high frequencies, the glass effectively observes a time-averaged temperature field, resulting in a steady thickness response. An intermediate regime exists between these two regimes, which gives rise to the observed resonant peak.

There is a clear worst location for the temperature disturbance to occur at $x_{\mathrm{loc}}=0.47$ (Figure $5 \mathrm{~b}$ ). Referring back to the steady state (Figure 3), we observe that this corresponds to location of the most rapid variations in the velocity of the glass. A temperature disturbance at this location results in large changes in the viscosity of the glass, which in turn affects the velocity and thickness profiles.

\subsection{Model Validation}

It is worth questioning whether the linearized theory accurately predicts the behaviour of the full nonlinear system, especially when the steady state is known to be unstable and large-amplitude responses are predicted, as in Figure 4(b). To validate our model we compare the results of our linear solver with those from a full numerical simulation. The full numerical simulation was performed using ANSYS Polyflow [2], within which the full Navier-Stokes equations coupled with an energy equation were implemented in $2 \mathrm{D}$. On the free surface the standard zero normal stress, zero tangential stress, and kinematic 
(a)

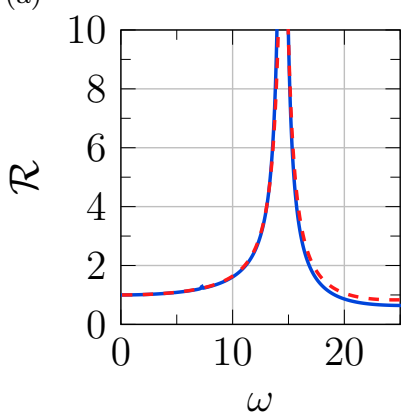

(b)

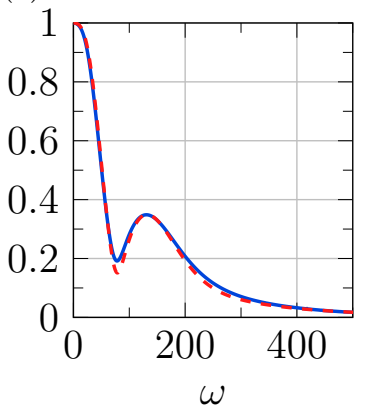

(c)

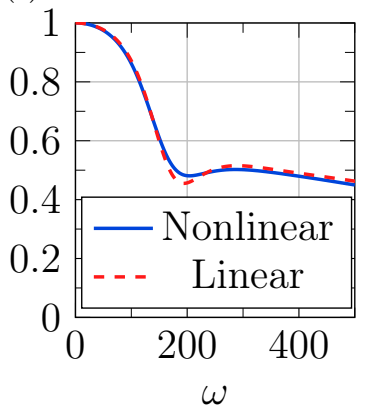

Fig. 6: Comparison of relative reponse amplitude $\mathcal{R}$ (defined in eqn. 10 ) predicted by the full Stokes equations with $\epsilon=0.01$ (solid) and by the linearized model (9) (dashed) for (a) feed/draw-speed disturbances in the isothermal case $(a=0)$, (b) feed-speed disturbances in the temperature-dependent case (c) draw speed disturbances in the temperature-dependent case. The parameter values in Table 1 are used with the exception of $b=0$.

conditions were applied as well as radiative and Newton-cooling conditions. The velocities at the inlet and outlet were set to be uniform and directed purely normal to the boundary of the computational domain and took the same user-defined value through the thickness of the glass. Additionally, the temperature at the inlet was prescribed (as in our reduced model) while the outlet condition was set to be given by a zero conductive heat flux.

We use the computational model to study the same three model scenarios as shown in Figure 4, setting a finite small disturbance amplitude $\epsilon=0.01$ for the nonlinear simulations. In Figure 6, we show the response to (a) a feed-speed disturbance in the isothermal case $(a=0)$; (b) a feed-speed disturbance with temperature effects included; and (c) a draw-speed disturbance with temperature effects included. We use the parameter values listed in Table11, except for $b$, which we set to be zero for simplicity when solving the full nonlinear model. We only compare for relatively low frequencies in the isothermal case because to resolve high frequencies requires an extremely high number of spatial points.

The simplified linearized model is found to approximate the value of $\mathcal{R}$ predicted by the full nonlinear simulation extremely well for both isothermal and temperature-dependent glass sheets. The results of our model validation provide confidence in the accuracy of the linear model in predicting the response of the full system, including the location and behaviour in the neighbourhood of large peaks in $\mathcal{R}$. We next use our linear model to investigate in detail the global effect on the relative response amplitude $\mathcal{R}$ of disturbances in the feed-speed,draw-speed and temperature. 

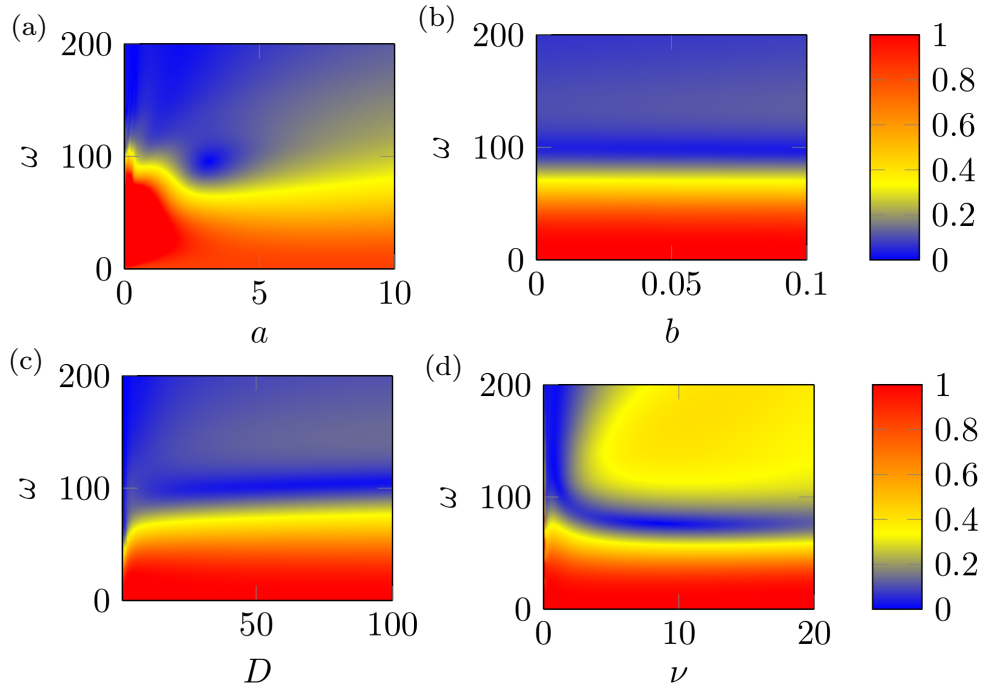

Fig. 7: Heatmap plots showing the dependence of the relative response amplitude $\mathcal{R}$ on the disturbance frequency $\omega$ and the system parameters (a) $a$, (b) $b$, , c) $D$ and (d) $\nu$, when subject to feed-speed disturbances. In each case, all parameters besides the one being varied are given by the values in Table 1 .

(a)

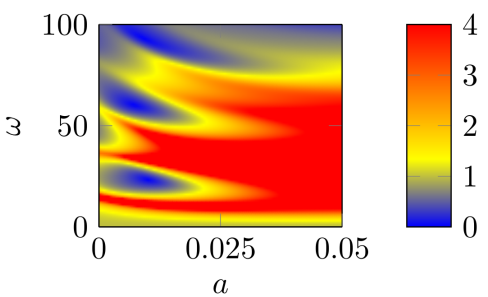

(b)

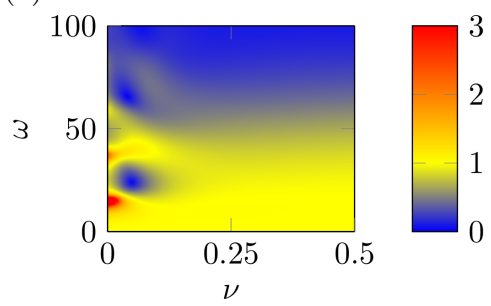

Fig. 8: Heatmap plots showing the dependence of the relative response amplitude $\mathcal{R}$ for small values of (a) $a$, and (b) $\nu$. In both cases, all other parameters are given by the values in Table 1 .

\subsection{Feed-speed disturbances}

Parameter sweeps for the case of feed-speed disturbances are plotted in Figure 7, where red regions correspond to large responses. We observe that the response $\mathcal{R}$ is largest at low frequencies. Since these low-frequency disturbances occur over long time scales their effects can be controlled relatively easily. The exceptions to this trend occur at extreme values of the process parameters. For example, as $D \rightarrow 1$, the changes in steady-state velocity and thickness during redraw are small, and the effect of perturbations is minimal (Figure $7 \mathrm{k}$ ). The detailed behaviour at small values of $a$ and $\nu$ is plotted in Figure 8. We observe, that as $a \rightarrow 0$ and/or $\nu \rightarrow 0$, we approach the isothermal case of a 
uniform-viscosity sheet, where the response is as shown in Figure 4(a), with a large peak close to $\omega \approx 14$ and a second peak at $\omega \approx 40$.

In Figure 7(a), as temperature dependence is strengthened by increasing $a$, we observe a decrease in response $\mathcal{R}$ at low frequencies $(\omega \lesssim 100)$ but a rise in $\mathcal{R}$ at higher frequencies $(\omega \gtrsim 100)$. This behaviour has the effect of spreading the response more evenly across frequencies and removing resonant peaks. If $\mathcal{R}$ is reduced to an acceptable value by this spreading then increasing the temperature dependence of the glass will aid in the production of more uniform glass sheets. However, if $\mathcal{R}$ is large over too broad a range of $\omega$, then increasing the temperature dependence of the glass will make it harder to control the thickness variations in the final product because many frequencies are being excited.

The response to perturbations appears particularly insensitive to variations in $b$, although it is important to note that the range over which $b$ is varied is smaller than the other parameters (Figure $7 \mathrm{~b}$ ). (The problem becomes more computationally challenging to solve for larger values of $b$.)

Beyond small values of $\nu$ we observe that the change in $\mathcal{R}$ as the frequency $\omega$ is increased is non-monotonic: $\mathcal{R}$ is first close to 1 , decreasing as approaches $\omega$ approaches $\omega \approx 90$ before increasing again (Figure $7 \mathrm{~d}$ ). It is possible to counteract the effect of low-frequency disturbances since they occur over a long time scale, but Figure 7(d) illustrates that high-frequency disturbances may also be a concern; this parameter sweep does not rule out the possibility that the $\mathcal{R}$ might increase further at even larger frequencies. A similar, although less extreme, phenomenon can be seen in the $\omega>100$ range as $D$ and $b$ are increased and for intermediate values of $a$. An asymptotic analysis to examine the behaviour at very high frequencies, where the numerical approach becomes prohibitively expensive, is carried out in 6 .

\subsection{Draw-speed disturbances}

In the isothermal case, draw-speed disturbances and feed-speed disturbances are equivalent. However, for a temperature-dependent scenario we observe in Figure 9 very different behaviour from the feed-speed perturbation response plotted in Figure 7. The most apparent difference is the apparently monotonic decrease in the response $\mathcal{R}$ with increasing $\omega$ for all parameter ranges considered in our sweep for draw-speed perturbations. Meanwhile, the range of low frequencies producing a large response (coloured red in Figure 9 increases with increasing $a, b, D$ or $\nu$. The wide band of excitable frequencies means that thickness variations cannot be controlled by targeting particular frequencies as they could in the feed-speed-perturbation case. The relative response amplitude $\mathcal{R}$ for draw-speed disturbances is also typically higher than that for feed-speed disturbances.

The size of the response $\mathcal{R}$ is insensitive to the value of $b$ for the range of $b$ considered (Figure 9b), and is also relatively insensitive to variations in both $a$ and $D$ except at very small values of those parameters (Figure 9a,c). 

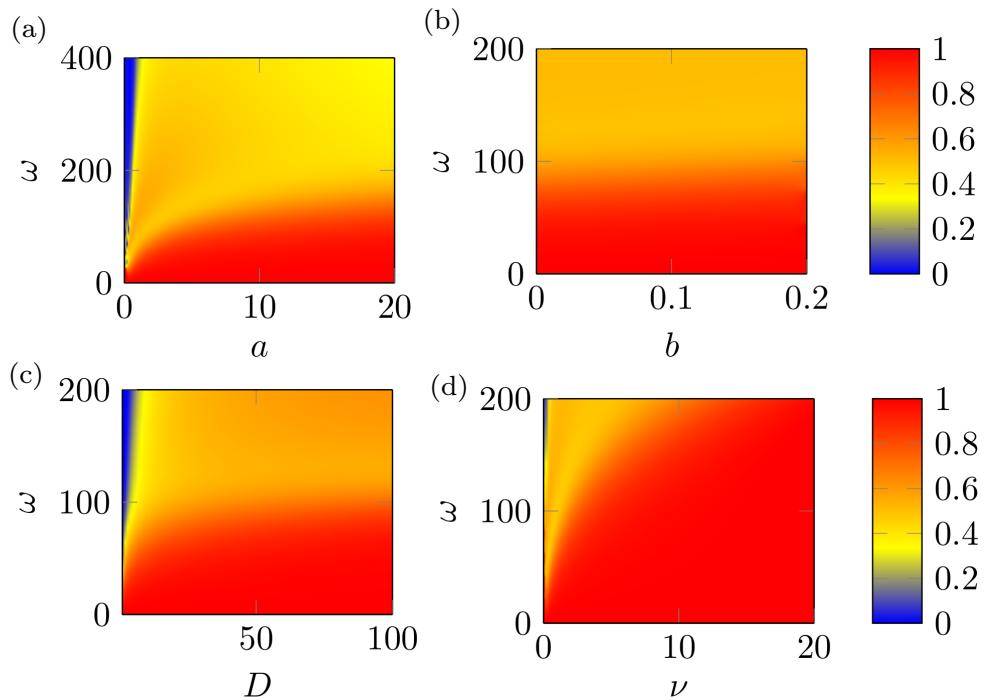

Fig. 9: Heatmap plots showing the dependence of the relative response amplitude $\mathcal{R}$ on the frequency $\omega$ and the system parameters (a) $a$, (b) $b$, (c) $D$ and (d) $\nu$, when subject to draw-speed disturbances.

\subsection{Temperature disturbances}

Finally, in Figure 10 we show the results of a parameter sweep for the case of disturbances to the furnace air temperature, with fixed location $x_{\text {loc }}=0.3$ and spread $\sigma^{2}=0.001$. If either $a$ or $\nu$ is zero, then the glass viscosity is not affected by the air temperature, and so the thickness variation caused by a temperature fluctuation must be zero. Nevertheless, Figure 10(a) shows that there is a region of large responses $\mathcal{R}$ for small values of $a$ and moderate $\omega$. Varying $b$, we observe in Figure $10(\mathrm{~b})$ a sharp transition from very small thickness variations when $b \lesssim 0.02$ to very large thickness variations when $b \gtrsim 0.05$. For the particular industrial regime described by Table 1 we have $b=0.022$ and the thickness variations remain relatively small for all $\omega$.

Figure 10 (c) shows that increasing the draw ratio $D$ leads to larger $\mathcal{R}$, since the final thickness decreases with increasing $D$. As in $\$ 5.2$, at low frequencies the system is in a pseudo-steady state, while at high frequencies the system instead sees an effectively steady time-averaged temperature distribution. It is therefore only in an intermediate band of frequencies that the temperature disturbance produces a large value of the response $\mathcal{R}$. While the range of potentially dangerous frequencies range is quite large, we can still identify a peak resonant frequency of $\omega \approx 100$ to avoid across most draw ratios.

For varying $\nu$, we also observe that the response is small at low frequencies, for the same reason as above (Figure $10 \mathrm{~d}$ ). However, at high frequencies the response is not significantly damped when $\nu$ is relatively large. Increasing $\nu$ dramatically increases the sensitivity of the viscosity to the temperature, 
(a)

3100

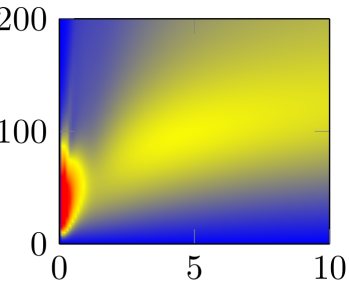

(c)

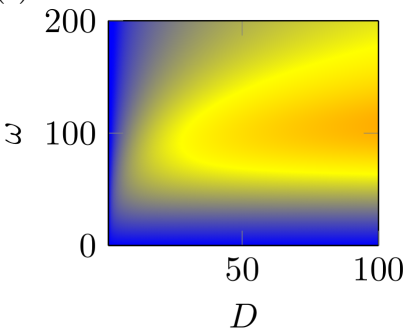

(b)

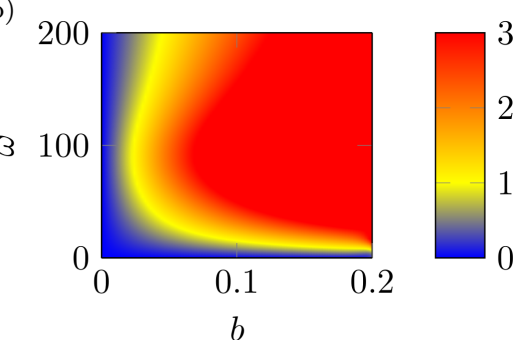

(d)

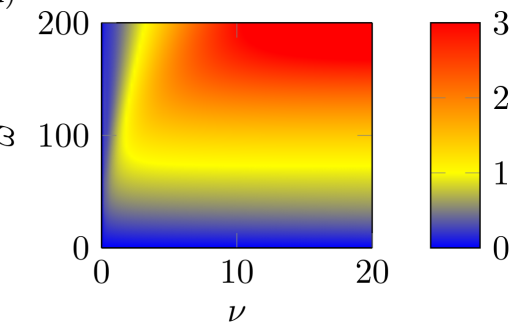

Fig. 10: Heatmap plots showing the dependence of the relative response amplitude $\mathcal{R}$ on the frequency $\omega$ and the system parameters (a) $a$, (b) $b$, (c) $D$ and $(\mathrm{d}) \nu$ when subject to temperature disturbances of the form $6 \mathrm{c}$ with $x_{\text {loc }}=0.3$.

so that even small deviations from a time-averaged temperature profile can contribute significantly to the viscosity of the glass and therefore to the final thickness variations.

\section{Asymptotic analysis for high frequency perturbations}

\subsection{Outline}

Our linearized model allows us to perform computationally cheap parameter sweeps to illustrate the system response to a wide range of perturbations. However, the numerical scheme becomes challenging to solve at large values of the perturbation frequency $\omega$. In this section we exploit the high-frequency limit to make further analytical progress and bypass the complications encountered in the numerics for the linearized model used so far. By obtaining the limiting behaviour of the response as $\omega \rightarrow \infty$, we can extend the results shown in Figures 7,10 and make sure that no further dangerous peaks can occur for higher values of $\omega$.

We find that the full solution in the large- $\omega$ limit is composed of a linear combination of a particular solution and a homogeneous solution. The first corresponds to a linear asymptotic expansion in the small parameter $1 / \omega$, which is analysed in 86.2 and the second corresponds to a WKBJ expansion, which is analysed in 86.3 By fitting the full solution to the boundary condi- 
tions, a leading-order approximation to the response is derived in 6.46 .5 and compared with our numerics in $\$ 6.6$.

\subsection{Particular solution}

We begin by seeking a solution composed of a linear expansion in the small parameter $1 / \omega$ :

$$
\begin{aligned}
\tilde{u}(x) & \sim \tilde{u}_{0}(x)+i \omega^{-1} \tilde{u}_{1}(x)+\ldots, \\
\tilde{h}(x) & \sim i \omega^{-1} \tilde{h}_{1}(x)+\omega^{-2} \tilde{u}_{2}(x)+\ldots, \\
\tilde{t}(x) & \sim i \omega^{-1} \tilde{t}_{1}(x)+\omega^{-2} \tilde{t}_{2}(x)+\ldots, \\
\tilde{F} & \sim \tilde{F}_{0}+i \omega^{-1} \tilde{F}_{1}+\ldots
\end{aligned}
$$

We substitute this expansion into 9a $-9 \mathrm{c}$ to find, at leading order in $\omega^{-1}$,

$$
\begin{aligned}
& \tilde{u}_{0}^{\prime}-h_{\mathrm{eq}} h_{1}=0, \\
& \tilde{u}_{0}^{\prime}+h_{\mathrm{eq}} u_{\mathrm{eq}}^{\prime}\left(\tilde{u}_{0}-\tilde{F}_{0}\right)=0, \\
& \tilde{u}_{0} T_{\mathrm{eq}}^{\prime}-h_{\mathrm{eq}} \tilde{t}_{1} T_{\mathrm{eq}}-a b \bar{T}_{a} f_{T}=0 .
\end{aligned}
$$

Using $u_{\mathrm{eq}} h_{\mathrm{eq}}=1$, we solve (14) to get

$$
\begin{aligned}
& \tilde{u}_{0}=\tilde{F}_{0}+\alpha_{0} h_{\text {eq }}, \\
& \tilde{h}_{1}=\alpha_{0} \frac{h_{\text {eq }}^{\prime}}{h_{\text {eq }}}, \\
& \tilde{t}_{1}=\frac{T_{\text {eq }}^{\prime}}{T_{\text {eq }}}\left(\tilde{F}_{0} u_{\text {eq }}+\alpha_{0}\right)-a b \bar{T}_{a} \frac{u_{\text {eq }}}{T_{\text {eq }}} f_{T},
\end{aligned}
$$

where $\alpha_{0}$ is a constant of integration. Both $\tilde{F}_{0}$ and $\alpha_{0}$ are to be determined later on by the application of the boundary conditions.

\subsection{Homogeneous solution}

We seek a homogeneous solution to 9 (with the inhomogeneities $\tilde{F}$ and $f_{T}$ set to zero) of WKBJ form:

$$
\begin{aligned}
& \tilde{u}(x)=\tilde{U}(x) \exp (i \omega \psi(x)), \\
& \tilde{h}(x)=\tilde{H}(x) \exp (i \omega \psi(x)), \\
& \tilde{t}(x)=\tilde{T}(x) \exp (i \omega \psi(x)),
\end{aligned}
$$

and make expansions of the form

$$
\begin{aligned}
\tilde{U}(x) & \sim \omega^{-2} \tilde{U}_{2}(x)+i \omega^{-3} \tilde{U}_{3}(x)+\ldots, \\
\tilde{H}(x) & \sim i \omega^{-1} \tilde{H}_{1}(x)+\omega^{-2} \tilde{H}_{2}(x)+\ldots, \\
\tilde{T}(x) & \sim i \omega^{-1} \tilde{T}_{1}(x)+\omega^{-2} \tilde{T}_{2}(x)+\ldots
\end{aligned}
$$


At leading order in $\omega^{-1}$, we obtain the eikonal equation

$$
\psi^{\prime}=-h_{\mathrm{eq}} .
$$

At next order, we obtain the system of ODEs

$$
\begin{aligned}
& \tilde{H}_{1}^{\prime}+\tilde{U}_{2} \psi^{\prime}=0, \\
& h_{\text {eq }} u_{\text {eq }}^{\prime}\left(\tilde{H}_{1}-\frac{\nu T_{\text {eq }}}{\left(T_{\text {eq }}-T_{\mathrm{c}}\right)^{2}} \tilde{T}_{1}\right)+\tilde{U}_{2} \psi^{\prime}=0, \\
& \tilde{T}_{1}\left(4 a T_{\text {eq }}^{4}+a b T_{\text {eq }}+T_{\text {eq }}^{\prime}\right)+\tilde{H}_{1} T_{\text {eq }}^{\prime}+T_{\text {eq }} \tilde{T}_{1}^{\prime}=0 .
\end{aligned}
$$

Equation $19 \mathrm{~b}$ gives $\tilde{U}_{2}$ in terms of $\tilde{H}_{1}, \tilde{T}_{1}$, and the steady-state solution. Substitution of $19 \mathrm{~b}$ into $19 \mathrm{a}$ and rearrangement of $19 \mathrm{c}$ gives

$$
\begin{aligned}
& \tilde{H}_{1}^{\prime}=u_{\mathrm{eq}}^{\prime} h_{\mathrm{eq}}\left(\tilde{H}_{1}-\frac{\nu T_{\mathrm{eq}}}{\left(T_{\mathrm{eq}}-T_{\mathrm{c}}\right)^{2}} \tilde{T}_{1}\right) \\
& \tilde{T}_{1}^{\prime}=-\frac{T_{\mathrm{eq}}^{\prime}}{T_{\mathrm{eq}}} \tilde{H}_{1}-\left(a b+4 a T_{\mathrm{eq}}^{3}+\frac{T_{\mathrm{eq}}^{\prime}}{T_{\mathrm{eq}}}\right) \tilde{T}_{1},
\end{aligned}
$$

which is a system of ODEs for $\tilde{H}_{1}$ and $\tilde{T}_{1}$.

\subsection{Boundary conditions}

The full solution is given by the sum of the particular and homogeneous expansions as

$$
\begin{aligned}
\tilde{u}(x) & \sim \tilde{u}_{0}(x)+i \omega^{-1} \tilde{u}_{1}(x)+\omega^{-2}\left(\tilde{u}_{2}(x)+\tilde{U}_{2}(x) \exp (i \omega \psi(x))\right)+\ldots, \\
\tilde{h}(x) & \sim i \omega^{-1}\left(\tilde{h}_{1}(x)+\tilde{H}_{1}(x) \exp (i \omega \psi(x))\right)+\ldots \\
\tilde{t}(x) & =i \omega^{-1}\left(\tilde{t}_{1}(x)+\tilde{T}_{1}(x) \exp (i \omega \psi(x))\right)+\ldots \\
\tilde{F} & =\tilde{F}_{0}+i \omega^{-1} \tilde{F}_{1}+\ldots
\end{aligned}
$$

We take $\psi(0)=0$, without loss of generality, so that the leading-order boundary conditions are

$$
\tilde{u}_{0}(0)=f_{\text {in }}, \quad \tilde{u}_{0}(1)=f_{\text {out }} .
$$

Applying (22) together with (5), we obtain

$$
\alpha_{0}=\frac{\left(f_{\text {in }}-f_{\text {out }}\right) D}{D-1}, \quad F_{0}=\frac{D f_{\text {out }}-f_{\text {in }}}{D-1} .
$$

At first order in $\omega^{-1}$ the boundary conditions are

$$
\tilde{u}_{1}(0)=0, \quad \tilde{H}_{1}(0)=-\tilde{h}_{1}(0), \quad \tilde{T}_{1}(0)=-\tilde{t}_{1}(0) .
$$


6.5 Asymptotic response amplitude

The leading-order asymptotic approximation for the relative response amplitude is given by

$$
\mathcal{R}=\omega^{-1}\left|\tilde{h}_{1}(1)+\tilde{H}_{1}(1) \exp (i \omega \psi(1))\right| .
$$

Here, $\tilde{h}_{1}(1)$ and $\tilde{H}_{1}(1)$ are constants (independent of $\omega$ ), so the amplitude of the response scales with $\omega^{-1}$, while oscillating as a function of $\omega$ according to the exponential term in $(25)$.

By substituting from (5) and (23) into $\sqrt{15}$, we find

$$
\tilde{h}_{1}(1)=-\frac{F_{\mathrm{eq}}\left(f_{\mathrm{in}}-f_{\mathrm{out}}\right)}{4(D-1) \mu\left(T_{\mathrm{eq}}(1)\right)},
$$

and

$$
\psi(1)=-\int_{0}^{1} h_{\mathrm{eq}}(x) \mathrm{d} x,
$$

where $F_{\text {eq }}$ and $h_{\text {eq }}(x)$ are given in (5). It therefore only remains to evaluate $\tilde{H}_{1}(1)$, by solving the linear system of ODEs 20 subject to the boundary conditions 24, which may be written out explicitly in the form

$$
\begin{aligned}
& \tilde{H}_{1}(0)=\frac{F_{\mathrm{eq}} D\left(f_{\mathrm{in}}-f_{\mathrm{out}}\right)}{4(D-1) \mu\left(T_{f}(0)\right)} \\
& \tilde{T}_{1}(0)=a b\left(1-\bar{T}_{a}\right) f_{\mathrm{in}}-\frac{a b \bar{T}_{a} f_{T}(0)}{T_{f}(0)} .
\end{aligned}
$$

For any given set of parameter values, we only have to solve two initial-value problems for the system (20), subject to the two sets of boundary conditions

$$
\begin{array}{ll}
H_{1}^{(1)}(0)=1, & T_{1}^{(1)}(0)=0 \\
H_{1}^{(2)}(0)=0, & T_{1}^{(2)}(0)=1 .
\end{array}
$$

The relevant contribution to the response 25 is then given by

$$
\begin{aligned}
\tilde{H}_{1}(1)=f_{\text {in }} & \left(\frac{F_{\mathrm{eq}} D H_{1}^{(1)}(1)}{4(D-1) \mu\left(T_{f}(0)\right)}+a b\left(1-\bar{T}_{a}\right) H_{1}^{(2)}(1)\right) \\
& -f_{\text {out }}\left(\frac{F_{\mathrm{eq}} D H_{1}^{(1)}(1)}{4(D-1) \mu\left(T_{f}(0)\right)}\right)-f_{T}(0)\left(\frac{a b \bar{T}_{a} H_{1}^{(2)}(1)}{T_{f}(0)}\right) .
\end{aligned}
$$

By considering in turn the terms proportional $f_{\text {in }}, f_{\text {out }}$ and $f_{T}$, we can separate out the response to feed-speed, draw-speed and temperature fluctuations 
as follows:

feed speed:

$$
\begin{aligned}
\mathcal{R} & \sim \omega^{-1} \mid \frac{F_{\mathrm{eq}}}{4(D-1) \mu\left(T_{\mathrm{eq}}(1)\right)} \\
& -\left(\frac{F_{\mathrm{eq}} D H_{1}^{(1)}(1)}{4(D-1) \mu\left(T_{f}(0)\right)}+a b\left(1-\bar{T}_{a}\right) H_{1}^{(2)}(1)\right) e^{i \omega \psi(1)} \mid
\end{aligned}
$$

draw speed: $\quad \mathcal{R} \sim \omega^{-1} \frac{F_{\text {eq }}}{4(D-1)}\left|\frac{1}{\mu\left(T_{\text {eq }}(1)\right)}-\frac{D H_{1}^{(1)}(1) e^{i \omega \psi(1)}}{\mu\left(T_{f}(0)\right)}\right|$,

$$
\text { temperature: } \quad \mathcal{R} \sim \omega^{-1} \frac{a b \bar{T}_{a} f_{T}(0)}{T_{f}(0)}\left|H_{1}^{(2)}(1)\right| .
$$

The leading-order response to temperature fluctuations, given by (30c), depends only on the inlet temperature perturbation $f_{T}(0)$. When $\sigma$ is small, $f_{T}(0)$ is effectively zero, apart from cases where $x_{\mathrm{loc}} \approx 0$ (for example, $f_{T}(0)<$ $10^{-18}$ when $\sigma^{2}=0.001$ and $\left.x_{\text {loc }}=0.3\right)$. For order 1 values of $x_{\text {loc }}$, we can therefore neglect $f_{T}(0)$ in $30 \mathrm{c}$, in which case thermal fluctuations have an insignificant influence on the response at this order in $\omega$. It is then necessary to proceed to higher order in $\omega$ in the asymptotic expansions to find a nontrivial approximation for the response to thermal fluctuations. By continuing the procedure outlined above up to order $\omega^{-2}$, for situations where $f_{T}(0)$ and $f_{T}(1)$ are negligibly small, we find that the asymptotic thermal response amplitude is given by

$$
\mathcal{R} \sim \omega^{-2} \frac{\tilde{F}_{1} F_{\mathrm{eq}}}{4}\left|\frac{D}{\mu\left(T_{\mathrm{eq}}(1)\right)}-\frac{H_{1}^{(1)}(1) e^{i \omega \psi(1)}}{\mu\left(T_{f}(0)\right)}\right|,
$$

where the first correction to the tension is given by

$$
\tilde{F}_{1}=\frac{a b F_{\mathrm{eq}} \nu \bar{T}_{a}}{4(D-1)} \int_{0}^{1} \frac{f_{\mathrm{T}}(x) \mathrm{d} x}{h_{\mathrm{eq}}(x)^{2} \mu\left(T_{\mathrm{eq}}(x)\right)\left(T_{\mathrm{eq}}(x)-T_{c}\right)^{2}} .
$$

When $\sigma$ is small, $f_{T}$ is approximately a delta-function and we can further approximate

$$
\tilde{F}_{1} \sim \frac{a b F_{\mathrm{eq}} \nu \bar{T}_{a}}{4(D-1) h_{\mathrm{eq}}\left(x_{\mathrm{loc}}\right)^{2} \mu\left(T_{\mathrm{eq}}\left(x_{\mathrm{loc}}\right)\right)\left(T_{\mathrm{eq}}\left(x_{\mathrm{loc}}\right)-T_{c}\right)^{2}} .
$$

We observe that, in the high-frequency limit, the most dangerous location for fluctuations in the air temperature is close to the inlet, such that $f_{T}(0)$ is maximised. For fluctuations in the interior of the heating zone, the influence on the thickness of the drawn sheet is much weaker, decaying like $\omega^{-2}$ instead of $\omega^{-1}$. Moreover, we can see that the maximum response to internal thermal fluctuations occurs when $x_{\text {loc }}$ is close to the minimum value of the denominator in equation $31 \mathrm{~b}$. 

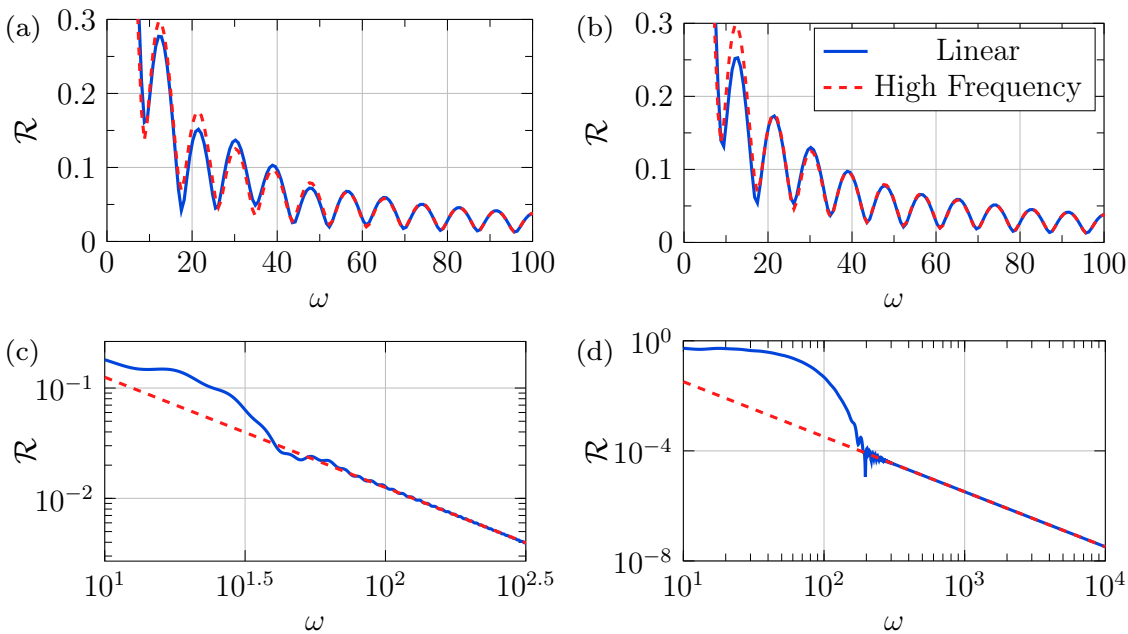

Fig. 11: Comparison of high-frequency asymptotic solution given by (30) with numerical solution of $(9)$ for $\nu=0.01$ and $D=2$ : (a) feed speed disturbances; (b) draw speed disturbances; (c) temperature disturbance with $x_{\text {loc }}=0.1$, $\sigma^{2}=0.01 ;(\mathrm{d})$ temperature disturbance with $x_{\mathrm{loc}}=0.45, \sigma^{2}=0.001$. All other parameter values are as in Table 1

\subsection{Results}

In Figure 11, we compare the response amplitude $\mathcal{R}$ predicted by the asymptotic approximations $(30)$ with that predicted by numerical solution of $\sqrt{9}$ for four cases of interest: (a) feed-speed perturbations, (b) draw-speed perturbations, (c) perturbations to the air temperature close to the inlet, and (d) perturbations to the interior air temperature. The predictions of the asymptotic analysis match closely with the numerical results for a sufficiently high frequency, $\omega$. As predicted by (30), the response to feed- or draw-speed fluctuations decays linearly with $\omega^{-1}$ with superimposed oscillations of approximately constant period. In Figure 11.(c), we impose a thermal fluctuation that is close to the inlet and relatively spread out, with $x_{\text {loc }}=0.1$ and $\sigma^{2}=0.01$, resulting in a non-negligible value of $f_{T}(0) \approx 2.876$. Consequently, we observe that the response amplitude decays like $\omega^{-1}$, with negligible oscillations for large $\omega$, in agreement with (30c). In Figure 11(d) we revert to a sharply peaked thermal fluctuation in the interior of the heating zone, with $x_{\text {loc }}=0.45$ and $\sigma^{2}=0.001$, so that $f_{T}(0)<10^{-42}$. In this case, we observe that the response to thermal fluctuations decays significantly more rapidly, like $\omega^{-2}$.

For the purposes of ensuring disturbances to the final thickness profile remain below a certain magnitude, the practitioner may employ 9 to determine dangerous low and mid-range frequencies, but can halt the numerical search at sufficiently large values of $\omega$ and rely on 30 thereafter. The asymptotic approximations require numerical integration of the ODE system (20), but 
this solution is very much simpler than for the full linearized problem (9). The frequency has been scaled out of $(20)$, so the problem does not become stiff when $\omega$ is large, and requires a less refined numerical grid to ensure accuracy and convergence. Furthermore, for a chosen set of parameter values, the problem (20) need only be solved twice, using the canonical boundary conditions (28), to obtain the leading-order response $\mathcal{R}$ for any (large) value of $\omega$.

\section{Conclusions}

We present a mathematical model to predict the response of a glass sheet undergoing redraw to fluctuations that arise during the manufacturing process. Specifically, we consider three sources of fluctuations that are common in the redraw process: the feed speed of the glass; the draw speed of the glass; and the ambient temperature profile of the surrounding air. These fluctuations arise from imperfections in the manufacturing equipment or to irregularities in the surroundings.

The response to a fluctuation may be determined by simulating the full redraw process numerically, but this is computationally challenging and timeconsuming. We derive a reduced model that exploits the fact that the perturbations are typically small. This provides a simplified linear model that is significantly simpler to solve, reducing computational times from days to minutes. The reduced model enables us to perform comprehensive parameter sweeps to identify the operating regimes that are most susceptible to instabilities. We consider the system response to four key parameters of interest: the draw ratio, the radiative Stanton number, the ratio of the Stanton number to the radiative Stanton number, and the strength of dependence of viscosity on temperature variations.

For high frequencies our linearized theory becomes more difficult to solve numerically. However, we are able to exploit the high-frequency limit to obtain a further reduced model. The resulting theory, comprising a combination of linearized and WKBJ solutions, is shown to predict the full system behaviour well and avoids the numerical complications encountered for the full linearized model. The asymptotic theory also provides estimates for the most dangerous locations of thermal fluctuations in the heating zone.

The resonant modes that we have observed in this work are reminiscent of those found in the so-called draw resonance. One proposed explanation for this phenomenon involves comparing the time scales involved in the different processes; with further investigation it may be possible to extend such ideas to provide additional physical intuition for our situation.

The model framework laid out here has been demonstrated to be robust and to predict the response to perturbations well. The parameter sweeps that we present here can be conducted for any given physical scenario. The model is simple to implement and we hope will provide an essential tool for the analysis of fluctuations in the redraw process. 


\section{References}

[1] Schott AG. Schott AG: Synthetic internal data on the glass redraw process (data is representative of typical values but has been altered to preserve confidentiality). Internal data (not available to public).

[2] ANSYS Polyflow Version 17.0.0.

[3] S. Burke. "This glass can bend in half without shattering (online video clip)." In: CNN Money http://edition.cnn.com/videos/cnnmoney/ 2016/03/18/bendable-glass-schott-burke-pkg.cnn-money/video /playlists/technology/ (2016).

[4] S d'Halewyu, Jean François Agassant, and Y Demay. "Numerical simulation of the cast film process". In: Polymer Engineering \& Science 30.6 (1990), pp. 335-340.

[5] T Dobroth and Lewis Erwin. "Causes of edge beads in cast films". In: Polymer Engineering 65 Science 26.7 (1986), pp. 462-467.

[6] Angiolo Farina et al. Mathematical Models in the Manufacturing of Glass: CIME Summer School, Montecatini Terme, Italy 2008. Springer, 2010.

[7] A. Filippov and Z. Zheng. "Dynamics and shape instability of thin viscous sheets". In: Physics of Fluids 22.2 (2010), p. 023601.

[8] D. Gelder. "The stability of fiber drawing processes". In: Industrial 8 Engineering Chemistry Fundamentals 10.3 (1971), pp. 534-535.

[9] T. Horvatitsch. "Ultra-thin glass." In: Schott Solutions 1/2016 (2016), pp. 6-11.

[10] P. D. Howell. "Extensional thin layer flows". PhD thesis. University of Oxford, 1994.

[11] J. C. Hyun et al. "Draw resonance in polymer processing: a short chronology and a new approach". In: Korea-Australia Rheology Journal 11.4 (1999), pp. 279-285.

[12] M. A. Matovich and J. R. A. Pearson. "Spinning a molten threadline. Steady-state isothermal viscous flows". In: Industrial \& Engineering Chemistry Fundamentals 8.3 (1969), pp. 512-520.

[13] M. R. Myers. "A model for unsteady analysis of preform drawing". In: AIChE Journal 35.4 (1989), pp. 592-602. DOI: 10.1002/aic.690350409

[14] D. O'Kiely. "Mathematical models for the glass sheet redraw process". PhD thesis. University of Oxford, 2017.

[15] D O'Kiely et al. "Edge behaviour in the glass sheet redraw process". In: Journal of Fluid Mechanics 785 (2015), pp. 248-269.

[16] A. G. Page et al. "Unraveling radial dependency effects in fiber thermal drawing". In: Applied Physics Letters 115.4 (2019), p. 044102. DOI: 10. 1063/1.5109469

[17] J. R. A. Pearson and M. A. Matovich. "Spinning a Molten Threadline. Stability". In: Industrial \& Engineering Chemistry Fundamentals 8.4 (1969), pp. 605-609. DOI: 10.1021/i160032a001.

[18] C. J. S. Petrie and M. M. Denn. "Instabilities in polymer processing". In: AIChE Journal 22.2 (1976), pp. 209-236. DOI: 10.1002/aic.690220202 
[19] M. Renardy. "Draw resonance revisited". In: SIAM Journal on Applied Mathematics 66.4 (2006), pp. 1261-1269.

[20] B. Scheid et al. "On the (de) stabilization of draw resonance due to cooling". In: J. Fluid Mech. 636 (2009), pp. 155-176.

[21] Yatish T Shah and JRA Pearson. "On the stability of nonisothermal fiber spinning-general case". In: Industrial \& Engineering Chemistry Fundamentals 11.2 (1972), pp. 150-153.

[22] David Silagy, Yves Demay, and J-F Agassant. "Study of the stability of the film casting process". In: Polymer Engineering \& Science 36.21 (1996), pp. 2614-2625.

[23] M. Taroni et al. "Asymptotic solutions of glass temperature profiles during steady optical fibre drawing". In: Journal of Engineering Mathematics 80.1 (2013), pp. 1-20. ISSN: 1573-2703. DOI: 10.1007/s10665-0139623-z.

[24] F. T. Trouton. "On the Coefficient of Viscous Traction and Its Relation to that of Viscosity". In: Proc. Roy. Soc. A 77.519 (1906), pp. 426-440. DOI: $10.1098 / \mathrm{rspa} .1906 .0038$.

\section{Acknowledgements}

This publication is based on work supported by the EPSRC Centre For Doctoral Training in Industrially Focused Mathematical Modelling (EP/L015803/1) in collaboration with Schott AG. SGM and DOK are grateful for funding from Schott AG and the Mathematical Institute, University of Oxford. IMG gratefully acknowledges support from the Royal Society through a University Research Fellowship. 